\title{
Glutathione Peroxidase cDNA Cloning and Expression in Soybean Roots under Heterodera glycines Infection
}

\author{
Fang Wang ${ }^{1,2}$, Yuxi Duan ${ }^{1}$, Lijie Chen ${ }^{1}$, Yuanyuan Wang ${ }^{1}$, Xiaofeng Zhu ${ }^{1} \& \mathrm{Wei}^{2}{ }^{3}$ \\ ${ }^{1}$ College of Plant Protection, Shenyang Agricultural University, Shenyang 110161, China \\ ${ }^{2}$ Heilongjiang Academy of Agricultural Sciences, Qiqihar Branch Academy, Qiqihar 161006, China \\ ${ }^{3}$ Husbandry Research Institute of Heilongiiang Province, 2 He Yi Street, Qiqihar 161005, China \\ Correspondence: Yuxi Duan, College of Plant Protection, Shenyang Agricultural University, Shenyang 110161, \\ China. Tel: 86-024-8845-4528. E-mail: duanyx64@163.com
}

Received: March 20, 2012 Accepted: April 1, 2012 Online Published: May 25, 2012

doi:10.5539/jas.v4n7p163 URL: http://dx.doi.org/10.5539/jas.v4n7p163

\begin{abstract}
A gene named GmGPX1 encoding glutathione peroxidase (GPX) was cloned and sequenced from soybean roots infested Heterodera glycines by RT-PCR (Reverse Transcription PCR), which is a crucial enzyme in plant cells regulating reactive oxygen species (ROX). The cDNA length of cloned gene was 693bp, flanked by a 5 '-untranslated region of $7 \mathrm{bp}$ and a 3 '-untranslated region of $185 \mathrm{bp}$, containing six exons and five introns. Genomic DNA fragment was located at Glycine max chromosome 5. The open reading frame of cDNA which encodes a polypeptide of 166 amino acid residues and protein molecular weight was $18375.8 \mathrm{Da}$, theoretical isoelectric point 6.59 . The deduced amino acid sequence showed about $99 \%$ and $64 \%$ homology with $G$ max putative PHGPX (XP_003532707.1) and Arabidopsis thaliana GPX (NP_564813.1), respectively. The expression profile of the GmGPX1 in Xiaoliheidou (G. max) under H. glycines infection, which generates oxidative stress, was analyzed. Real time PCR analysis revealed that the GmGPX1 mRNA levels were increased stabilized from 1.3 to 1.47 times after exposure to $H$. glycines from $12 \mathrm{~h}$ to $48 \mathrm{~h}$, and reduced to 1.07 times at $72 \mathrm{~h}$ comparing with non-inoculation control. These results suggest that the GmGPX1 gene is induced by H. glycines at early stage in soybean roots and play an important role in removing oxidative damage.
\end{abstract}

Keywords: soybean, glutathione peroxidase, expression pattern, soybean cyst nematode

\section{Introduction}

In plant cells, aerobic metabolisms particularly those involving in photosynthesis and respiration result in the generation of reactive oxygen species (ROS). Those ROS, such as superoxide radicals, hydroxyl radicals, or hydrogen peroxide, can damage biological molecules, including nucleic acid, lipids, and proteins. In order to protect against the oxidation stress, plants in vivo have developed several enzymatic and nonenzymatic systems (Grene, 1995). GPXs (glutathione peroxidase) play a critical role in enzymatic systems to detoxify hydrogen peroxide and protect plant cells against oxidative stress. Their principal activity is thought to catalyze the reduction of $\mathrm{H}_{2} \mathrm{O}_{2}$ and lipid hydroperoxide to water and alcohol (Bowler et al., 1992). GPX requires an ascorbate (AsA) or a glutathione (GSH) as substrate (Mittler, 2002). The first study of GPXs associated with catalytic activity and encoding gene originates from animal (Ursini et al., 1985). In 1992, the first cDNA homology to mammalian selenium-dependent GPXs is cloned from Nicotiana sylvestris leaves (Criqui et al., 1992). Since then, several homologous GPX cDNAs have been isolated from Citrus sinensis, Arabidopsis thaliana, Brassica campestris, pinacia olerae, Helianthus annuus, Lycopersicon esculentum, Pisum sativum, Oryza sativa and Chinese cabbage (Holland et al., 1993; Sugimoto \& Sakamoto, 1997; Eshdat et al., 1997; Sugimota et al., 1997; Roeckel-Drevet et al., 1998; Depege et al., 1998; Mullineaux et al., 1998; Li et al., 2000; Jung et al., 2002). Furthermore, researches find that the activity of GPXs in plant is lower than those in mammalian because of Cys catalytic sites in plant, whereas high reactivity of catalytic sites selenium-dependent Cys in mammalian (Faltin et al., 1998; Holland et al., 1993). In addition, analyses of GPXs mRNA expression level in plants present a stable level increase under different environmental constraints, such as pathogen infections, high salt, metal stress, mechanical stimulation, aluminum toxicity (Criqui et al., 1992; Holland et al., 1993; Sugimoto \& Sakamoto, 1997; Depege et al., 1998). 
In the previous study, we constructed a suppression subtractive hybridization - cDNA library, which soybean cyst nematode (Heterodera. glycines) induces Glycine max gene differentially expressed, and screened an EST fragment. This EST shares $98 \%$ similarity with soybean sequence (NM_001248911) by BLASTn, $86 \%$ similarity with Arachis hypogaea sequence (DQ889534, glutathione peroxidase 1 mRNA). So it is presumed that the EST is belonged to family member of glutathione peroxidase. In order to confirm and explicit function of the EST, we cloned the full-length ORF using reverse transcription PCR and analyzed the expression characteristic for the further study.

\section{Materials and Methods}

\subsection{Plant Materials and Soybean Cyst Nematode Preparation}

H. glycines were originated by single-cyst descent maintained on G. max cv. Liaodou 15 in the greenhouse of Nematology Institute of Northern China. The population of $H$. glycine genotype was identified race 3 according to Golden system (Golden et al., 1970).

The G. $\max \mathrm{cv}$. Xiaoliheidou (National code ZDD1412) used in the experiments was incompatible with race 3 determined in previous studies (Liu et al., 1994). The seeds were soaked in $10 \% \mathrm{NaClO}$ to remove the pathogenic bacteria, and then washed with sterile water for $5 \mathrm{~min}$. Put seeds in the petri dishes loaded with moisture sterile filter paper, germinated at $25^{\circ} \mathrm{C}$ for $2 \mathrm{~d}$ in illuminating incubator. Transplant the seeds to plastic pot until the length of radical were $1 \mathrm{~cm}$. Seedlings watered daily were grown in plastic pot (one plant per pot) containing 50\% sterile soil and 50\% sand. After 2 weeks seedling, plants were inoculated with a suspension of $4000 \mathrm{~J} 2$ (second-stage juveniles) by pipetting into three holes $(13 \mathrm{~cm}$ diameter $\mathrm{x} 12 \mathrm{~cm}$ depth) around the roots.

The plants were challenged with nematodes were classified into one group, whereas plants without nematodes infection were used as control group. Root tips ( $2 \mathrm{~cm}$ length to tip) of five seedlings were sampled at $0,12,24$, 48 , and $72 \mathrm{~h}$ post-inoculation, individually, for both groups.

\subsection{Total RNA Isolation and cDNA Synthesis}

Total RNAs were extracted following protocol of SV Total RNA Isolation Kit (Promega, USA). The quality and quantity of extracted RNA was determined, and equal amounts of root RNA per time point were pooled from the five sampled plants for each group. $1 \mu \mathrm{g}$ of total RNA from samples was reverse transcribed according to introduction of Promega Reverse Transcription System (Promega, USA). Reaction system included $\mathrm{MgCl}_{2} 4 \mu \mathrm{l}$, reverse transcription $10 \mathrm{X}$ buffer $2 \mu \mathrm{l}$, dNTP mixture $2 \mu \mathrm{l}$, recombinant RNase in $0.5 \mu \mathrm{l}$, AMV reverse transcriptase $0.6 \mu \mathrm{l}$, Oligo $(\mathrm{dT})_{15}$ primer $1 \mu \mathrm{l}$, total RNA1 $\mu \mathrm{g}$, nuclease-free water to a final volume of $20 \mu \mathrm{l}$. Incubate the reaction at $42^{\circ} \mathrm{C}$ for 60 minutes, then heat samples at $95^{\circ} \mathrm{C}$ for 5 minutes to inactivate the AMV reverse transcriptase and prevent it from binding to the cDNA. Store the cDNA at $-20^{\circ} \mathrm{C}$ until use.

\subsection{GmGPXI cDNA Cloning}

According to reference sequence NM_001248911 in NCBI, the specific primers of gene GmGPX1 were designed as followed: sense: 5'-GGACACTATGGCCACCTCAAG-3', anti-sense primer: 5'-CACACAATAATAAGTACACAATCTC-3'. The transcribed synthesized cDNA were used as templates for PCR amplification. Reaction system included cDNA template 1.5 $\mu 1,2 \mathrm{X}$ Taq PCR Master Mix 12.5 $\mu$ (Tiangen, China), sense primer $1 \mu \mathrm{l}(10 \mathrm{mmol} / \mathrm{L})$, anti-sense $1 \mu \mathrm{l}(10 \mathrm{mmol} / \mathrm{L})$, double distilled $\mathrm{H}_{2} \mathrm{O}$ to total volume $25 \mu 1$. Reaction program was done as followed: pre-denaturation at $95^{\circ} \mathrm{C}$ for $2 \mathrm{~min}$; denaturation at $95^{\circ} \mathrm{C}$ for $30 \mathrm{~s}$, annealing at $57^{\circ} \mathrm{C}$ for $30 \mathrm{~s}$, and extension at $72^{\circ} \mathrm{C}$ for $1 \mathrm{~min}$, followed by 30 cycles; extension at $72^{\circ} \mathrm{C}$ for $10 \mathrm{~min}$.

PCR product was separated by $1.2 \%(\mathrm{v} / \mathrm{w})$ agarose gel electrophoresis, purified with DNA gel extraction kit (Tiangen, China), connected to pGEM -T Easy Vector with the size of 3105bp (Promega, USA), and transformed to competent Escherichia.coli DH5 $\alpha$ cells by heat strike at $42^{\circ} \mathrm{C}$. White-blue selection and colonies PCR confirmation were carried out, followed by the plasmid extraction from over-night cultured lysate using plasmid extraction kit (Tiangen, China). Recombinant restriction analysis was done using EcoR I restriction enzyme, then examined on $1.2 \%(\mathrm{v} / \mathrm{w})$ agarose gel electrophoresis. The plasmid containing the goal gene was sequenced.

\subsection{Expression Analysis by Real-time Quantitative PCR}

Real-time quantitative PCR was used to determine GmGPX1 mRNA expression level. Root tips of Xiaoliheidou were harvested at 12, 24, 36, 48 and $72 \mathrm{~h}$ from both treatments (inoculation and non-inoculation group). First-strand cDNA was synthesized by $1 \mu \mathrm{g}$ total RNA from each sample using the Reverse Transcription System (Promega, USA) following the manufacturer's protocol. Gene-specific primers were designed by using Primer Premier 5.0 software. Actin 11 (Accession number BW652479) was used as reference gene for normalizing the transcript profiles. Primers of actin 11 sense: 5'- CGGTGGTTCTATCTTGGCATC-3', anti-sense: 5'- 
GTCTTTCGCTTCAATAACCCTA-3'; primers of GmGPX1 sense: 5'- CAAGGCTGAGTTTCCCGTT-3', anti-sense: 5'- ATACCATCCCCAAAGAGTCC-3'. The PCR reactions were prepared contained 10ul SYBR Premix Ex TaqTM II (Takara, China), $1 \mu$ l forward and reverse gene-specific primers $(10 \mu \mathrm{M}), 1.5 \mu \mathrm{l}$ of the five-fold diluted reverse transcribed cDNA, and $6.5 \mu$ l double distilled $\mathrm{H}_{2} \mathrm{O}$. The thermal cycling protocol entailed activation of Hot Start Taq DNA polymerase at $95^{\circ} \mathrm{C}$ for $10 \mathrm{~min}$, followed by 40 amplification cycles with denaturation at $94^{\circ} \mathrm{C}$ for $10 \mathrm{~s}$, primer annealing at $57^{\circ} \mathrm{C}$ for $30 \mathrm{~s}$ and extension at $72^{\circ} \mathrm{C}$ for $30 \mathrm{~s}$. The real-time PCR data were calibrated following the $2^{-\Delta \Delta}{ }^{\mathrm{Ct}}$ method for relative quantification of abundance (Livak \& Schmittgen, 2001). Real-time quantitative PCR was performed on a Mx 3000P platform (Stratagene, Santa Clara, CA).

\subsection{Sequences Analysis}

Homologous comparison was carried out using BLAST at http://blast.ncbi.nlm.nih.gov/; ORF and amino acid sequence were determined at http://www.ncbi.nlm.nih.gov/gorf/orfig.cgi; number and position of exons were predicted at http://www.ncbi.nlm.nih.gov/IEB/Research/Ostell/Spidey/; amino acid sequence composition, molecular weight of protein, isoelectric point and other physicochemical characteristic were predicted at http://web.expasy.org/protparam/. Sequences alignment was done by DNAMAN6.0 software; a neighbor-joining (NJ) phylogenetic tree of GmGPX1 with other GPX from plant was constructed using MEGA4 software.

\section{Results and Analysis}

\subsection{GmGPX1 cDNA Cloning}

The cDNA from G. max Xiaoliheidou infected with H. glycines was used as PCR template. PCR amplified a sequence with the approximate size of $700 \mathrm{bp}$ (Figure 1). Purified this fragment, followed by blue-white screening and restriction analysis with EcoR I. Examined the fragment on $1.2 \%(\mathrm{v} / \mathrm{w})$ agarose gel electrophoresis The result showed the target gene has been successfully connected with vector (Figure 2). The vector was extracted and sequenced using T7 promoter primer and SP6 promoter primer by ABI PRISM ${ }^{\mathrm{TM}} 3730 \mathrm{XL}$ DNA sequencer. The sequenced product was BLASTn in NCBI, which the cDNA fragment has $99 \%$ similarity with soybean sequence (XP_003532707.1), so was named as GmGPX1.

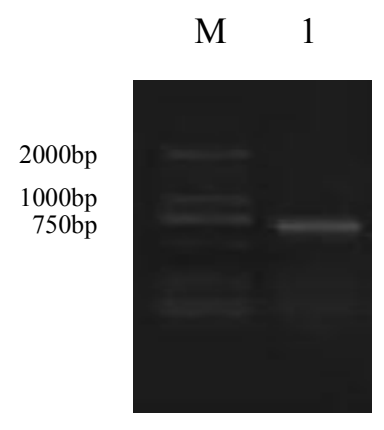

Figure 1. PCR product of GmGPX1

Note: M. DL2000 Marker; 1. PCR product from cDNA

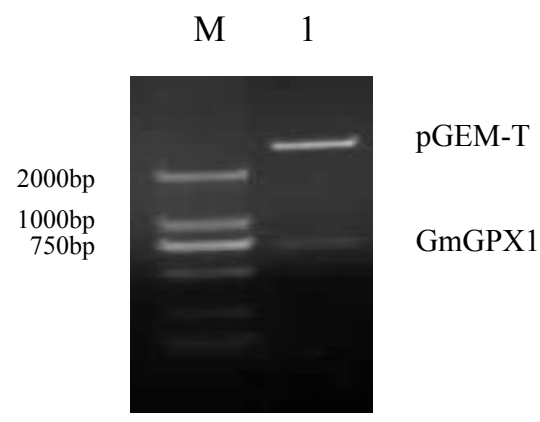

Figure 2. Analysis of recombinant plasmids digested with EcoR I

Note: M. DL2000 Marker; 1. PCR product of recombinant plasmids digested with EcoR I 3.2 GmGPX1 Bioinformatic Analysis

3.2.1 Amino Acid and Homologous Comparison

The cloned cDNA full length of GmGPX1 was $693 \mathrm{bp}$, located at soybean genome 5 chromosome, flanked by a 
5 '-untranslated region of $7 \mathrm{bp}$ and a 3 '-untranslated region of $185 \mathrm{bp}$, including one poly A structure at $536 \mathrm{bp}$, a full-length open reading frame of $501 \mathrm{bp}$, encoding 166 amino acids. We attempted to obtain the genomic sequence of GmGPX1 and the result showed 6 exons using Spidey software. ExPASy software predicted the molecular weight was $18375.8 \mathrm{Da}$, protein molecular formula $\mathrm{C}_{829} \mathrm{H}_{1293} \mathrm{~N}_{211} \mathrm{O}_{252} \mathrm{~S}_{4}$, theory isoelectric point 6.59.

Homologous amino acid sequences of GmGPX1 were searched using BLASTp at http://blast.ncbi.nlm.nih.gov/. Use DNAMAN and MEGA4 to build amino acid alignment (Figure 3) and neighbor-joining (NJ) phylogenetic tree (Figure 4). The figure shows that amino acid sequence of GmGPX1 sharing the highest homology with $G$ max (XP_003532707.1), which similarity is 99\%; then followed by Lotus japonicas (AAP69867.1), Medicago truncatula (XP_003630520.1), Nicotiana tabacum (BAB16430.1), Arachis hypogaea (ACF74299.1). Lowest homology is with Arabidopsis thaliana (NP_564813.1), which similarity is 64\%.
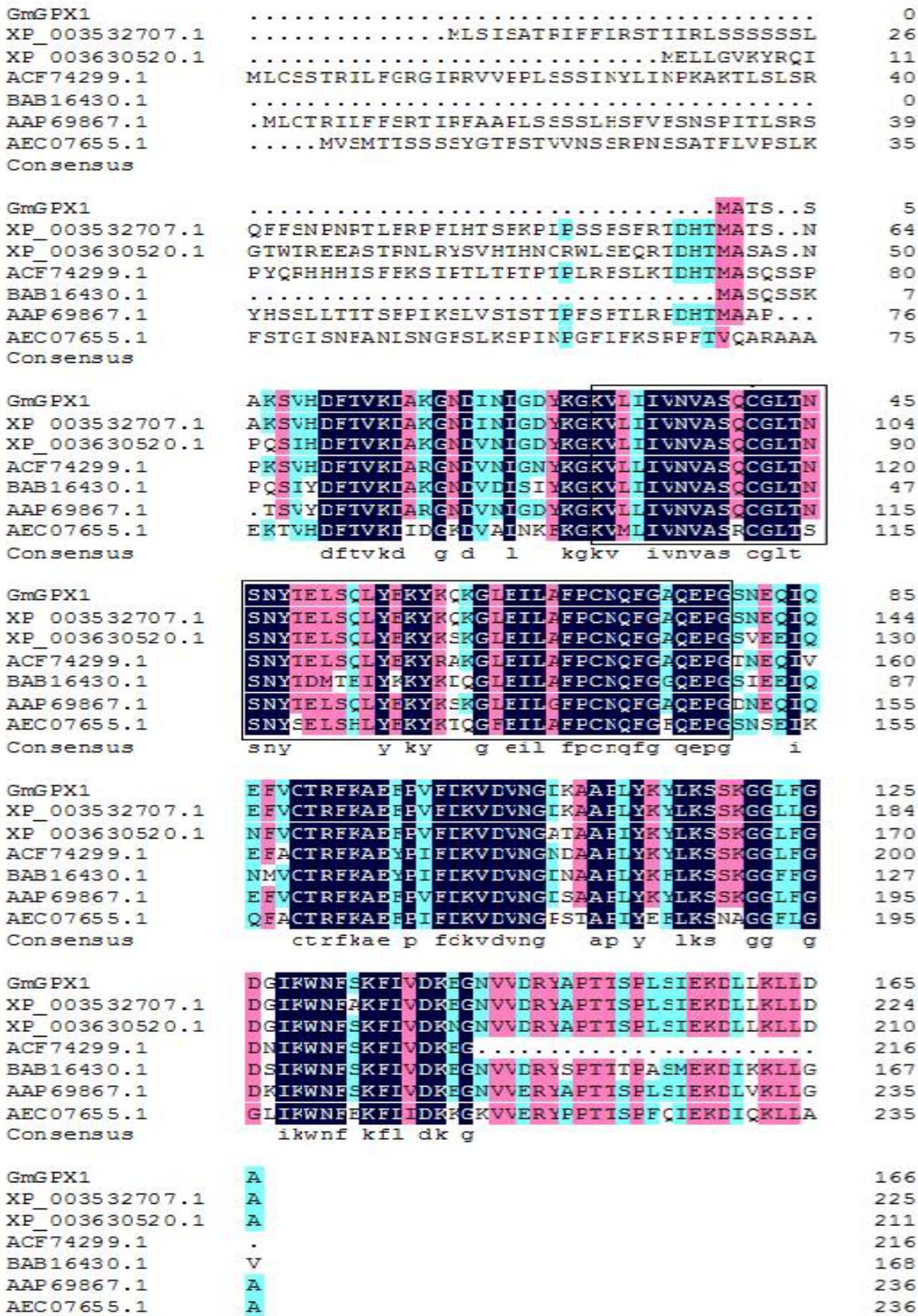

DGIRWNESKFIVDKEGNVVDRYAPTISEL SIERLIIRLID

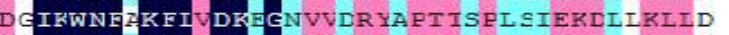
DGRNATI

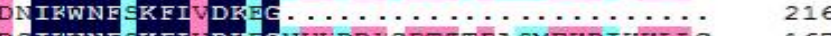
DSIRWNFSKFIVDKAGNVVDRYSPTITFASMERDIKRIIG 167 DKIRWNESKFIVDKEGNVVERYAPIISPLSIERDIVRIIG 235 GI IRWNABKFIIDKKGKVVERYPPTISPFC्रIERDICRIIA ikwnf $\mathrm{kfl} d \mathrm{~kg}$

$$
235
$$

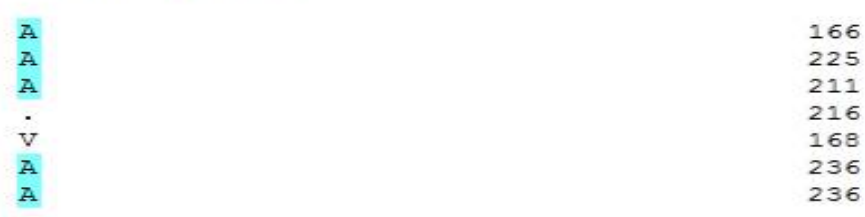

Figure 3. Amino acid alignment among GmGPX1, XP_003532707.1 (Glycine max), XP_003630520.1 (Medicago truncatula), ACF74299.1 (Arachis hypogaea), BAB16430.1 (Nicotiana tabacum), AAP69867.1 (Lotus japonicus), NP_564813.1 (Arabidopsis thaliana) 
Note: 1 . Black, pink and blue boxes represent $100 \%, 75 \%$ and $50 \%$ similarity, respectively

2. Catalytic triad of GmGPX1 were marked with triangles

3. The motif of PHGPX from GmGPX1 and other plant GPX
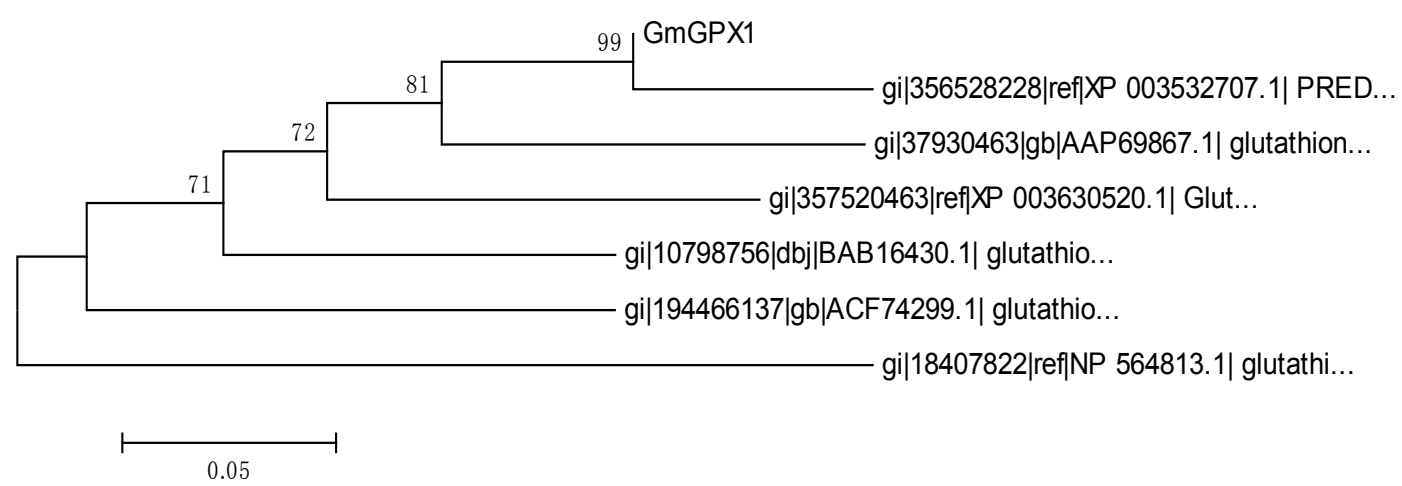

Figure 4. Phylogenetic tree of GmGPX1 with other GPXs from plant

Note: Glycine max (XP_003532707.1), Lotus japonicus (AAP69867.1), Medicago truncatula (XP_003630520.1), Nicotiana tabacum (BAB16430.1), Arachis hypogaea (ACF74299.1), Arabidopsis thaliana (NP_564813.1)

\subsubsection{Structure Domain Analysis}

The structure domain of gene GmGPX1 encoding protein was analyzed by CDD search in NCBI and SWISS MODEL (http://swissmodel.expasy.org). The result appears that GmGPX1 shares high homology with GSH peroxidas (at residues 9-117), belonged to thioredoxin _like superfamily (at residues 7-166), including three residues of catalytic triad, $\mathrm{Cys}^{41}, \mathrm{Gln}^{76}, \operatorname{Trp}^{130}$; six dimer interface sites (Fig.5). Multisequences comparision of GPX families proteins was done using software online http://prosite.expasy.org/cgi-bin/prosite/, obtained the motif of probable phospholipid hydroperoxide glutathione peroxidase (PHGPX) motif, the pattern as followed: K-x(1,2)-L-x(0,1)-I-V-N-V-A-S-x-C-G-[LM]-T-N-S-N-Y-T-[DE]-[LM]-[NST]-[EQ]-[IL]-Y-x-[KR]-Y-[KR]-[A DQS]-x-G-L-E-I-L-[AG]-F-P-C-N-Q-F-G-[ADG]-[EQ]-E-P-G.

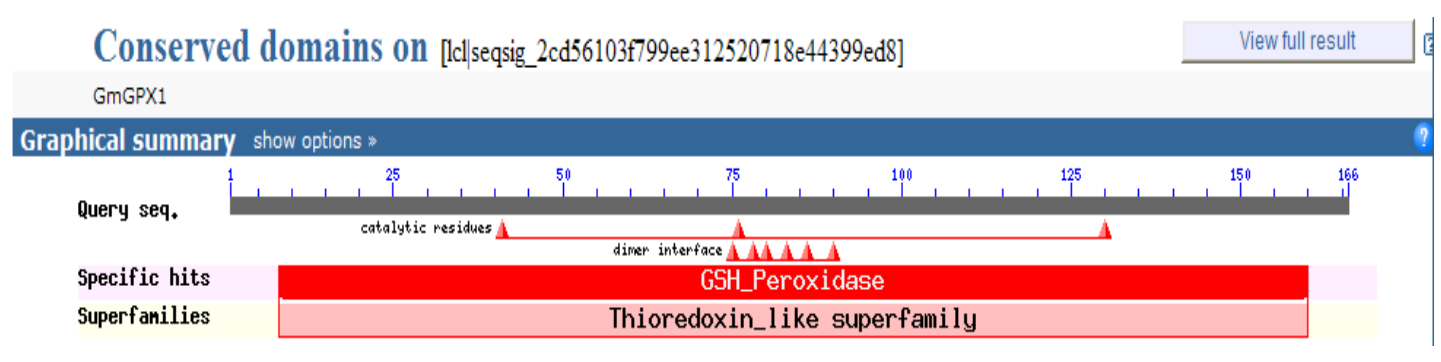

Figure 5. Conserved domain of GmGPX1 protein predicted by CDD software

\subsubsection{GmGPX1 Protein Hydrophobicity Analysis}

Hydrophobicity analysis of GmGPX1 protein was carried out using ProtScale programm at http://web.expasy.org/protscale/. The analyzed content was Hphob. / Kyte \& Doolittle, following Kyte \& Doolittle method. The $\mathrm{X}$ axis is represented amino acid sequence position; $\mathrm{Y}$ axis shows amino acid scale, which includes positive value representing hydrophobicity; negative value representing hydrophilic; peptide with higher hydrophobicity located at protein interior, peptide with higher hydrophilic located at protein surface. As shown in Figure. 6, there is typical hydrophobic region near N-terminal of GmGPX1 protein, located at residues $30-40$. However, residues $20,60,80,105,115,145,150$ are belong to high hydrophilic region. It is can be inferred that GmGPX1 shares a strong hydrophobicity and is a soluble protein. 


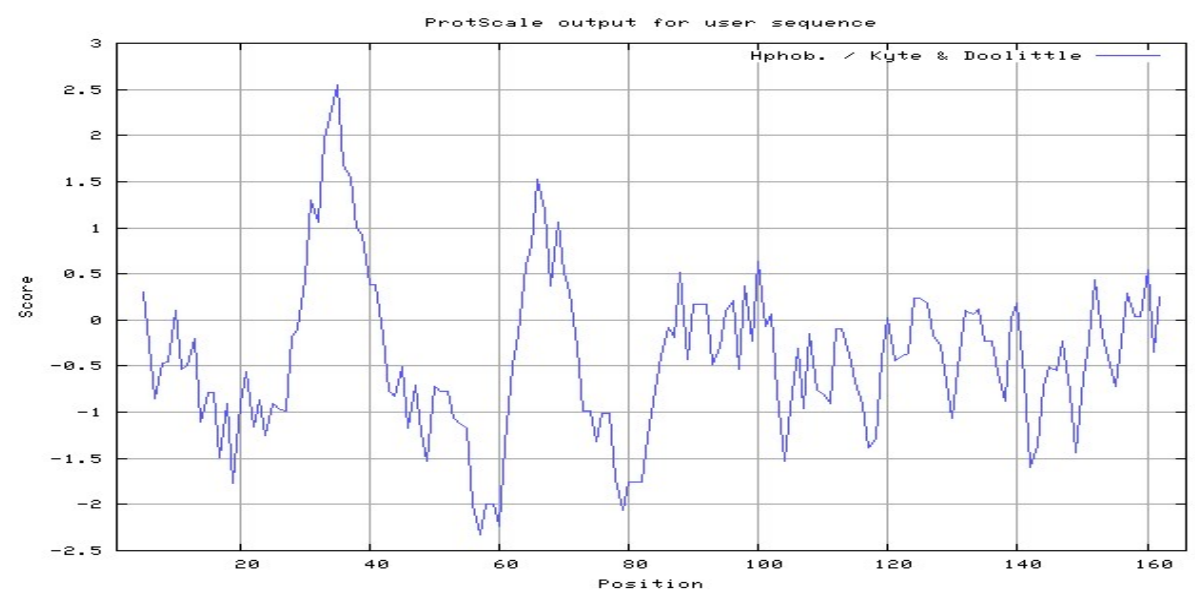

Figure 6. Hydrophobicity profile of GmGPX1 protein

\subsubsection{Active Sites and Subcellular Localization}

The active sites of GmGPX1 amino acid sequence were predicted using software at

https://www.predictprotein.org/. The active sites include two N-glycosylation site, residues at $47-50$ (NYTE) and $131-134$ (NFSK); three protein kinase C phosphorylation site, residues at 5 - 7 (SAK), $13-15$ (TVK) and 118 -120 (SSK); two casein kinase II phosphorylation site, residues at 8 - 11 (SVHD) and $13-16$ (TVKD); four $\mathrm{N}$-myristoylation site, residues at $19-24$ (GNDINL), $42-47$ (GLTNSN), 121 -126 (GGLFGD),127 - 132 (GIKWNF); glutathione peroxidases active site, residues at 29 - 44 (GKVLIIVNVASQCGLT); glutathione peroxidases signature 2, residues at $66-73$ (LAFPCNQF). The subcellular localization of GmGXH1 was predicted to be at chloroplast or mitochondria in plants by using LOCkey method, with $83 \%$ reliability. The protein of GmGXH1 is non-secreted and non-nuclei protein.

\subsection{Expression Analysis of GmGPX1 in Root Tips}

To evaluate the expression changes of GmGPX1 in soybean root tissues before and after $H$. glycines infection, we extracted RNAs from soybean root tissues with infection and those uninfected at different intervals as templates for real-time PCR. As shown in Figure 7and 8, the main parameter analysis in real-time PCR shows that the amplification plots of the reference gene actin 11 and target gene GmGPX1 represent standard S pattern; the amplification turned into index growth at 19 cycles; the peak of dissociation curve is single. Those indicate that the extracted samples with high quality, specific primers, there is no primer dimer or other non-specific products, reference gene and target gene share similar Tm value.

The mRNA expression level of GmGPX1 were up-regulated after infection from $12 \mathrm{~h}$ to $72 \mathrm{~h}$, with approximately $1.30,1.44,1.38,1.47$ and 1.07-fold compared to uninfected controls at different intervals, individually. The up-regulated expression was more apparent from $12 \mathrm{~h}$ to $48 \mathrm{~h}$, and then a slight declined (Figure 9). The entire period represented a stable up-regulated level. This result indicates that GmGPX1 is induced to be expressed after H.glycines infection.

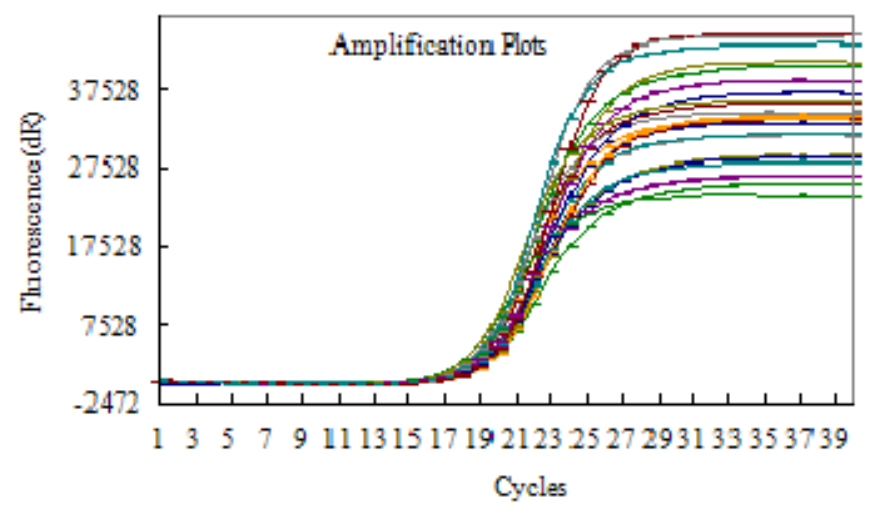

Figure 7. The amplification plots of the reference gene actin11 and target gene GmGPX1 


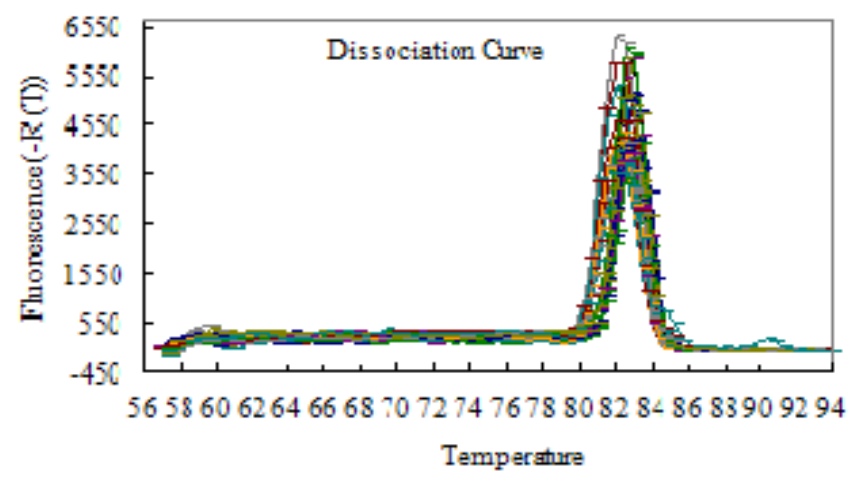

Figure 8 . The dissociation curve of the reference gene actin11 and target gene GmGPX1
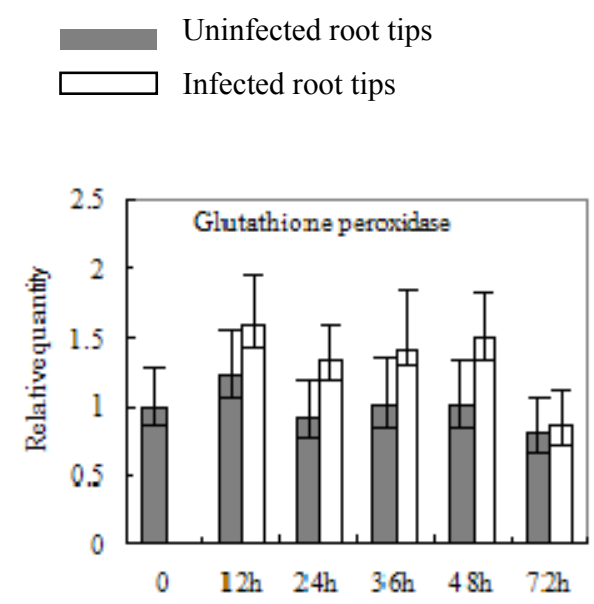

Figure 9. Relative quantity expression of GmGPX1 during 0-72h under inoculation and non-inoculation

\section{Discussion}

Glutathione peroxidases (GPXs) are a group of enzymes that are encoded by multiple genes. According to subcellular location, amino acid sequence, and conjuncted substrate, GPXs can be divided into several different isozymes. A family of seven genes encoding glutathione peroxidase has been identified in genome of Arabidopsis thaliana, named as AtGPX1-AtGPX8, respectively, which are located at the cytosol, chloroplasts, mitochondria, peroxisomes, and apoplast (Rodriguez et al., 2003). These genes are expressed ubiquitously and are regulated by abiotic stresses through diverse signaling pathways (Rodriguez et al., 2003). In mostly studies, the expression or activity of GPX is generally up-regulated in response to stress. However, there are some exceptions to this pattern. So this demonstrates that the function of these enzymes in plants is not completely the same. Two GPX isoforms increases in barley (Hordeum vulgare) under osmotic or methyl viologen-induced stress, a third GPX isoform (HVGPH3) is down-regulated under these conditions (Churin et al., 1999). Transcripts encoding a glutathione peroxidase (accession no. BE587404) are rapidly induced in response to low temperature stress, but other six AtGPXs haven't shown response to low temperature. This indicates that AtGPXs gene family is regulated by multiple signaling pathways at abiotic stresses (Rodriguez et al., 2002). Miao et al. (2006) finds that AtGPX3 might play dual and distinctive roles in $\mathrm{H}_{2} \mathrm{O}_{2}$ homeostasis, acting as a general scavenger and specifically relaying the $\mathrm{H}_{2} \mathrm{O}_{2}$ signal as an oxidative signal transducer in ABA and drought stress signaling. In plant, GPXs gene family contain multiple members, some members serving as enzymes are involved in aerobic metabolism and defense response at different evolutionary period and environmental stresses, while other members play an important role in multiple signaling transduction pathways.

As predicted that gDNA of GmGPX1 included 6 exons and 5 introns, this is the same as seven members in Arabidopsis thaliana AtGPX families and the predicted soybean GPX gene (XM_003532659) in NCBI. Comparing mRNA sequences of GmGPX1 and XM_003532659 to their genome, individually, it could find that 
Exon1 and Exon6 of GmGPX1 are longer than those of soybean GPX gene (XM_003532659), the length of other four exons are the same for both sequences. Analysis of structure and function of GmGPX1, it was showed that the GmGPX1 protein contains the motif of probable phospholipid hydroperoxide glutathione peroxidase (PHGPX), three conserved catalytic triads domain and three conserved Cys. GmGPX1 is belonged to non-secreted, non-nuclei protein by biological information analysis, the same as AtGPX1.

The soybean cyst nematode is an obligated sedentary endoparasite. Infective second-stage juveniles penetrate host roots and migrate intracellular where establishes feeding site. Over the course of movement and feeding site instruction, nematode causes cellular damage and stress, thereby induce the host defense response and wound-related responses. Plant cells produce superoxide $\left(\mathrm{O}_{2}{ }^{-}\right)$and its dismutation product, hydrogen peroxide $\left(\mathrm{H}_{2} \mathrm{O}_{2}\right)$, both of which are toxic to nematode parasite. $\mathrm{H}_{2} \mathrm{O}_{2}$ is accumulated not only in the developing syncytim cell walls, but also in cells surrounding developing syncytia and cells which are neither in contact with the nematode nor with the syncytium, especially in cells neighboring hypersensitivity reaction. In many plants, oxidative burst is the first defense response to nematode invasion(Baker \& Orland, 1995; Lamb \& Dixon, 1997). Reactive oxygen species as signal molecular that control and regulate biological process, such as cell death, abiotic stress, pathogen reaction, and defense pathway (Desikan et al., 2001; Knight \& Knight, 2001). In this study, the expression of GmGPX1 was increased gradually from $12 \mathrm{~h}$ to $48 \mathrm{~h}$ after inoculation. This indicates that after inoculation $12 \mathrm{~h}$, nematodes invasion causes the host cells damage or necrosis, resulting in cellular $\mathrm{H}_{2} \mathrm{O}_{2}$ increase, eventually the expression of GmGPX1 is up-regulated. Following the slow accumulation of $\mathrm{H}_{2} \mathrm{O}_{2}$, the expression of GmGPX1 is gradually increased. This may be presumed that invasion and movement of juveniles during 12-48h inoculation could result in an increased production of $\mathrm{H}_{2} \mathrm{O}_{2}$. There is a report that the more widespread accumulation of $\mathrm{H}_{2} \mathrm{O}_{2}$ is first apparent 12h after inoculation with Melodoigyne incognita and a heavy accumulation of $\mathrm{H}_{2} \mathrm{O}_{2}$ in the intercellular spaces in giant cells after 24h in resistant tomato roots (Melillo et al., 2006). At $72 \mathrm{~h}$ after inoculation, it began to decrease to 1.07 times comparing with non-infection control. This may be that fewer plant tissues are damaged at $72 \mathrm{~h}$. The infective juveniles migrate in root vascular tissues and initiate the development of a feeding site after a suitable cell selected during approximately 2 days after inoculation. Another reason should be that over production of $\mathrm{H}_{2} \mathrm{O}_{2}$ is also harmful to plant cell in vivo, so a mechanism that equilibrium the $\mathrm{H}_{2} \mathrm{O}_{2}$ level is activated, resulting in the expression of GmGPX1 decreased. This study shows that GmGPX1 is playing an important role in plant resistance reaction and remove the oxidative damage induced by soybean cyst nematode infection.

\section{References}

Baker, C. J., \& Orland, E. W. (1995). Active oxygen in plant pathogenesis. Annual Review Phytopathology, 33, 299-321.

Bowler, C., Montagu, M. V., \& Inzé, D. (1992). Superoxide dismutases and stress tolerance. Annual Review of Plant Physiology and Plant Molecular Biology, 43, 83-116.

Cho, M. J., \& Lee, S. Y. (2002). A Chinese cabbage cDNA with high sequence identity to phospholipid hydroperoxide glutathione peroxidases encodes a novel isoform of thioredoxin-dependent peroxidase. Journal of Biological Chemistry, 277, 12572-12578.

Churin, Y., Schilling, S., \& Borner, T. (1999). A gene family encoding glutathione peroxides homologues in Hordeum vulgare (barley). FEBS Letters, 459, 33-38.

Criqui, M. C., Jamet, E., Parmentier, Y., Marbach, J., Durr, A., \& Fleck, J. (1992). Isolation and characterization of a plant cDNA showing homology to animal glutathione peroxidases. Plant Molecular Biology, 18, 623-627.

Depege, N., Drevet, J., \& Boyer, N. (1998). Molecular cloning and characterization of tomato cDNAs encoding glutathione peroxidase-proteins. European Journal of Biochemistry, 253, 445-451.

Desikan, R., Mackerness, S. A. H., Hancock, J. T., \& Neill, S. J. (2001). Regulation of the Arabidopsis transcriptosome by oxidative stress. Plant Physiology, 127, 159-172.

Eshdat, Y., Holland, D., Faltin, Z., \& Ben-Hayyim, G. (1997). Plant glutathione peroxidases. Physiologia Plantarum, 100, 234-240.

Faltin, Z., Camoin, L., Ben-Hayyim, G., Perl, A., Beeor-Tzahar, T., Strosberg, A. D., Holland, D., \& Eshdat, Y. (1998). Cysteine is the presumed catalytic residue of Citrus sinensis phospholipids hydroperoxide glutathione peroxidase over-expressed under salt stress. Physiology Plant, 104, 741-746.

Golden, A. M., Epps, J. M., Riggs, R. D., Duclos, L. A., Fox, J. A., \& Bernard, R. L. (1970). Terminology and 
identity of infraspecific forms of the soybean cyst nematode (Heterodera glycines). Plant Disease Report, $54,544-546$.

Grene, R. (2002). Oxidative stress and acclimation mechansisms in plants. In: Someville C K, Meyerwitz E M, The Arabidopsis Book . American Society of Plant Biologist, Rockville, 1-19.

Holland, D., Ben-Hayyim, G., Faltin, Z., Camoin, L., Strosberg, A. D., \& Eshdat, Y. (1993). Molecular characterization of salt-stress-associated protein in citrus: protein and cDNA sequence homology to mammalian glutathione peroxidase. Plant Molecular Biology, 21, 923-927.

Jung, B. G., Lee, K. O., Lee, S. S., Chi, Y. H., Jang, H. H., Kang, S. S., Lee, K., Lim, D., Yoon, S. C., Yun, D. J., Inoue, Y., Knight, H., \& Knight, M. R. (2001). Abiotic stress signalling pathways: specificity and cross-talk. Trends in Plant Science, 6, 262-267.

Lamb, C., \& Dixon, R. A. (1997). The oxidative burst in plant disease resistance. Annual Review Plant Physiology Molecular Biology, 48, 251-75.

Li, W. J., Feng, H., Fan, J. H., Zhang, R. Q., Zhao, N. M., \& Liu, J. Y. (2000). Molecular cloning and expression of a phospholipid hydroperoxide glutathione peroxidase homolog in Oryza sativa. Biochimica et Biophysica Acta, 1493, 225-230.

Liu, W. Z., Hong, Q. C., Liu, Y., \& Duan, Y. X. (1994). Application of interorganismal genetics concept in grouping soybean genes resistant to soybean cyst nematode. Soybean Science, 1, 1-4. (in Chinese)

Livak, K. J., \& Schmittgen, T. D. (2001). Analysis of relative gene expression data using real-time quantitative PCR and the 2- ${ }^{-} \Delta^{\mathrm{Ct}}$ method. Methods, 25, 402-408.

Melillo, M. T., Leonetti, P., Bongiovanni, M., Castagnone-Sereno, P., \& Bleve-Zacheo, T. (2006). Modulation of reactive oxygen species activities and $\mathrm{H}_{2} \mathrm{O}_{2}$ accumulation during compatible and incompatible tomato-root-knot nematode interactions. New Phytologist, 170, 501-512.

Miao, Y. C., Dong, L., Wang, P. C., Wang, X. C., Chen, J., Miao, C., \& Song, C. P. (2006). An Arabidopsis glutathione peroxidase functions as both a redox transducer and a scavenger in abscisic acid and drought stress responses. The Plant Cell, 18, 2749-2766.

Mittler, R. (2002). Oxidative stress, antioxidants and stress tolerance. Trends in Plant Science, 7, 405-410.

Mullineaux, P. M., Karpinski, S., Jiménez, A., Cleary, S. P., Robinson, C., \& Creissen, G. P. (1998). Identication of cDNA encoding plastid-targeted glutathione peroxidase. The Plant Journal, 13, 375-379.

Rodriguez Milla, M. A., Butler, E., Huete, A. R., Wilson, C. F., Anderson, O., \& Gustafson, J. P. (2002). EST-based gene expression analysis under aluminum stress in rye (Secale cereale L.). Plant Physiology, 130, 1706-1716.

Rodriguez Milla, M. A., Maurer, A., Huete, A. R., \& Gustafson, J. P. (2003). Glutathione peroxidase genes in Arabidopsis are ubiquitous and regulated by abiotic stresses through diverse signaling pathways. Plant Journal, 36, 602-615.

Roeckel-Drevet, P., Gagne, G., De Labrouhe, T. D., Dufaure, J. P., Nicolas, P., \& Drevet, J. R. (1998). Molecular characterization organ distribution and stress-mediated induction of two glutathione peroxidase-encoding mRNAs in sunflower (Helianthus annuus). Physiologia Plantarum, 103, 385-394.

Sugimota, M., Furui, S., \& Suziki, Y. (1997). Molecular cloning and characterization of a cDNA encoding putative phopholipid hydroperoxide glutathione peroxidase from spinach. Bioscience Biotechnology Biochemistry, 61, 1379-1381.

Sugimoto, M., \& Sakamoto, W. (1997). Putative phospholipid hydroperoxide glutathione peroxidase gene from Arabidopsis thaliana induced by oxidative stress. Genes \& Genetic Systems, 72, 311-306.

Ursini, F., Maiorino, M., \& Brigelius-Flohé, R. (1995). Diversity of glutathione peroxidases. Methods in Enzymology, 252, 38-53.

Ursini, F., Maiorino, M., \& Gregolin, C. (1985). The selenoenzyme phosphlipid hydroperoxide glutathione peroxidase. Biochemistry Biophysics Acta, 839, 62-70. 